\title{
Correction: An oncogenic viral interferon regulatory factor upregulates CUB domain-containing protein 1 to promote angiogenesis by hijacking transcription factor lymphoid enhancer-binding factor 1 and metastasis suppressor CD82
}

Wan Li · Qingxia Wang · Xiaoyu Qi · Hongmei Lu • Yuheng Chen · Jiale Shi · Fei Wang · Ziyu Wang • Yao Lu • Zhongmou Lu $\cdot$ Qin Yan $\cdot$ Cong Wang $\cdot$ Shou-Jiang Gao $\cdot$ Chun Lu

Published online: 14 September 2020

(c) The Author(s), under exclusive licence to ADMC Associazione Differenziamento e Morte Cellulare 2020

Correction to: Cell Death \& Differentiation

https://doi.org/10.1038/s41418-020-0578-0
The original version of this Article did not acknowledge Yao Lu as a co-author. This has now been corrected in both the PDF and HTML versions] of the Article. 